\title{
Numerical Modeling of Turbulent Convective Heat Transfer for Supercritical Pressure Fluids Cooled in Horizontal Tubes
}

\author{
Yufei Mao, Fei Cao, Qingjun Liu, Wei Qi, Hong Xiao and Tianyu Zhu \\ College of Mechanical and Electrical Engineering, Hohai University, PR China
}

For corresponding: yufmao@126.com

Keywords: supercritical pressure, convective heat transfer, cooling tube, boundary layer.

\begin{abstract}
Modeling of the turbulent convective heat transfer to supercritical pressure fluids in horizontal circular tubes is achieved through an integral approach, and a traditional mixing length turbulence model is employed into the numerical scheme. Based on this model, heat transfer of supercritical carbon dioxide cooled in circular tubes was investigated numerically. The effects of mass flux, pressure, heat flux and tube diameter on heat transfer coefficient were simulated, and the simulation results were then compared with the experimental data. It is shown that the present model can provide fast and accurate predictions for the heat transfer behavior in the turbulent boundary layer of supercritical fluid flows under cooling conditions.
\end{abstract}

\section{Introduction}

Due to its environmental advantages and certain attractive thermal characteristics, Carbon dioxide $\left(\mathrm{CO}_{2}\right)$ as a promising alternative refrigerant has received considerable attention for many years. In order to optimize the coefficient of performance of tans-critical $\mathrm{CO}_{2}$ refrigeration system, turbulent convective heat transfer of supercritical $\mathrm{CO}_{2}$ cooled in horizontal circular tubes was investigated by a few researchers. Yoon et al. [1] carried out experiments in a tube with inner diameter of $7.73 \mathrm{~mm}$, and discussed the effects of mass flux and pressure on heat transfer. Dang and Hihara [2] performed experiments in a range of inner diameter $1 \sim 6 \mathrm{~mm}$, system pressure 8 10 MPa, mass flux 200 1200 $\mathrm{kg} \cdot \mathrm{m}^{-2} \cdot \mathrm{s}^{-1}$ and heat flux $6 \sim 33 \mathrm{~kW} \cdot \mathrm{m}^{-2}$, and the effects of different parameters on turbulent convective heat transfer were discussed in detail. In a later paper [3], Dang and Hihara applied four turbulence models to simulate the heat transfer of supercritical $\mathrm{CO}_{2}$ in tubes under both heating and cooling conditions, and the low Reynolds number $k-\varepsilon$ model by Jones and Launder showed the best agreement with the experimental data. Li et al. [4] conducted experiments and numerical simulations using semi-circular channels with a hydraulic diameter of $1.16 \mathrm{~mm}$ under both heating and cooling conditions, and concluded that for a given mass flux with a moderate heat flux in either heating or cooling mode, heat transfer is enhanced greatly in the pseudo-critical region where the specific heat increases greatly. The former investigations provide the incentive and basis for further research to find out the heat transfer mechanism to turbulent flow of supercritical fluids in cooling tubes.

At supercritical pressures, the thermophysical properties of a fluid undergo dramatic variations throughout the pseudo-critical region, which leads to the difficulty in numerical modeling for turbulent convective heat transfer. Unlike heat transfer in the heating mode, the deterioration in heat transfer and the ' $\mathrm{M}$ '-shape profile of radial velocity could not happen in the cooling mode. So it is reasonable that the boundary layer theory can be applied to supercritical fluid flows under cooling conditions. The objective of present study is to develop a theoretical model of heat transfer to supercritical fluids flowing circular cooling tubes, by which to simulate the behavior of turbulent heat transfer with strong variable-property effects. The simulation results were compared with the available experimental data for validation.

\section{Mathematical Model}

Governing equations. For pipe flow, the local conservation equations are integrated in an elemental volume of radius $r$ and thickness $\delta x$ (see Figure 1 and 2). The flow is assumed to be 
'quasi-developed' (Tanaka et al. [5]), in which the velocity profile is accelerated in a uniform proportion and the local pressure gradient $\mathrm{d} p / \mathrm{d} x$ is constant. Figure 1 shows the force balance in the elemental volume. According to Newton's second law, the equation of motion is yielded as follows:

$$
\tau=\left(\mu+\mu_{\mathrm{t}}\right) \frac{\partial u}{\partial y}=\frac{r}{R} \tau_{\mathrm{w}}+\frac{r}{2}\left(\rho_{\mathrm{b}} u_{\mathrm{b}}^{2}-\rho_{\mathrm{e}} u_{\mathrm{e}}^{2}\right) \frac{1}{u_{\mathrm{b}}} \frac{\mathrm{d} u_{\mathrm{b}}}{\mathrm{d} x}
$$

Where $\tau$ is the turbulent shear stress, $u$ is the fluid velocity, $R$ is the tube radius, $x$ is the axial direction, $y$ is the distance from the wall $(y=R-r), \mu$ is the dynamical viscosity of fluid, $\rho$ is the density of fluid; the subscripts $\mathrm{t}, \mathrm{w}, \mathrm{b}$ and $\mathrm{m}$ mean the turbulence, wall, bulk and elemental volume condition, respectively. Using $\xi$ as the intermediate variable, the $\rho_{\mathrm{e}}, \rho_{\mathrm{b}}, u_{\mathrm{e}}$ and $u_{\mathrm{b}}$ are calculated as:

$$
\rho_{\mathrm{e}}=\frac{2}{r^{2}} \int_{0}^{r} \rho \xi \mathrm{d} \xi, \rho_{\mathrm{b}}=\frac{2}{R^{2}} \int_{0}^{R} \rho \xi \mathrm{d} \xi, u_{\mathrm{e}}=\frac{\int_{0}^{r} \rho u \xi \mathrm{d} \xi}{\int_{0}^{r} \rho \xi \mathrm{d} \xi}, u_{\mathrm{b}}=\frac{\int_{0}^{R} \rho u \xi \mathrm{d} \xi}{\int_{0}^{R} \rho \xi \mathrm{d} \xi}
$$

The turbulent viscosity is calculated by the Van Driest's mixing length formulation [6]:

$$
\mu_{\mathrm{t}}=\rho l_{\mathrm{m}}^{2}\left|\frac{\partial u}{\partial y}\right|, l_{m}=0.4 y\left[1-\exp \left(-\frac{y^{+}}{26}\right)\right], y^{+}=\frac{\sqrt{\rho \tau_{\mathrm{w}}}}{\mu} y
$$

Figure 2 shows the energy balance in the elemental volume. Assuming the local enthalpy gradient $\partial h / \partial x$ is constant, the equation of energy is yielded as follows:

$q=-\left(\lambda+\lambda_{\mathrm{t}}\right) \frac{\partial T}{\partial y}=\left(1-\frac{y}{R}\right) \frac{\rho_{\mathrm{e}}}{\rho_{\mathrm{b}}} \frac{u_{\mathrm{e}}}{u_{\mathrm{b}}} q_{\mathrm{w}}$

Where $q$ is the heat flux, $T$ is the fluid temperature, and $\lambda$ is the thermal conductivity of fluid.

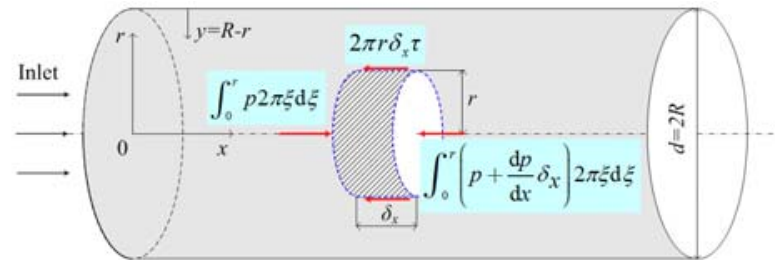

Fig. 1 Elemental volume for force balance

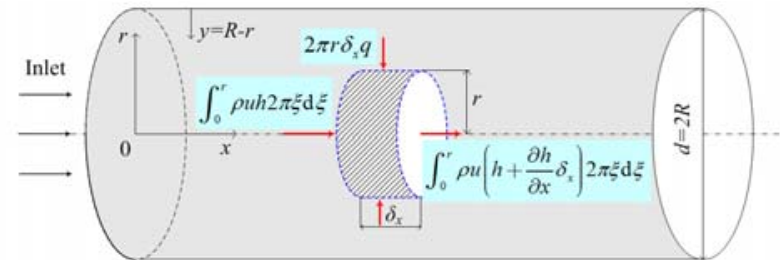

Fig. 2 Elemental volume for energy balance

Dimensionless governing equations. To solve the motion equation (1) and energy equation (4), the dimensionless method is used. Introducing the dimensionless variables:

$$
\eta=\frac{r}{R}, Y=\frac{y}{R}=1-\eta, U=\frac{\mu_{\mathrm{b}} u}{\tau_{\mathrm{w}} d}, \Theta=\frac{T_{\mathrm{w}}-T}{q_{\mathrm{w}} d / \lambda_{\mathrm{b}}}
$$

Where $d$ is the tube diameter, $d=2 R$. The motion and energy equations become:

$$
\left\{\begin{array}{l}
\frac{\partial U}{\partial Y}=0.5 \frac{\mu_{\mathrm{b}}}{\mu} \frac{1-Y}{1+\frac{\mu_{\mathrm{t}}}{\mu}}\left[1+\frac{\beta_{\mathrm{b}} q_{\mathrm{w}} U_{\mathrm{b}} R e_{\mathrm{b}}}{c_{p \mathrm{~b}} G}\left(1-\frac{\rho_{\mathrm{e}}}{\rho_{\mathrm{b}}} \frac{U_{\mathrm{e}}^{2}}{U_{\mathrm{b}}^{2}}\right)\right] \\
\frac{\partial \Theta}{\partial Y}=0.5 \frac{\lambda_{\mathrm{b}}}{\lambda} \frac{1-Y}{1+\frac{\mu_{\mathrm{t}}}{\mu} \frac{P r}{P r_{\mathrm{t}}}} \frac{\rho_{\mathrm{e}}}{\rho_{\mathrm{b}}} \frac{U_{\mathrm{e}}}{U_{\mathrm{b}}}
\end{array}\right.
$$

Where $G$ is the mass flux of fluid, $c_{p}$ is the isobaric specific heat capacity of fluid, $\beta$ is the volume expansivity of fluid, $R e$ is the Reynolds number $(R e=G d / \mu)$. The turbulent Prandtl number $P r_{\mathrm{t}}$ is set to be 1 . The boundary conditions are: $Y=0, U=0, \Theta=0$. The $\rho_{\mathrm{e}}, \rho_{\mathrm{b}}, U_{\mathrm{e}}$ and $U_{\mathrm{b}}$ are calculated as:

$$
\rho_{\mathrm{e}}=\frac{2}{\eta^{2}} \int_{0}^{\eta} \rho \xi \mathrm{d} \xi, \rho_{\mathrm{b}}=2 \int_{0}^{1} \rho \xi \mathrm{d} \xi, U_{\mathrm{e}}=\frac{\int_{0}^{\eta} \rho U \xi \mathrm{d} \xi}{\int_{0}^{\eta} \rho \xi \mathrm{d} \xi}, U_{\mathrm{b}}=\frac{\int_{0}^{1} \rho U \xi \mathrm{d} \xi}{\int_{0}^{1} \rho \xi \mathrm{d} \xi}
$$

The mixing length turbulence model in the dimensionless form is 


$$
\frac{\mu_{\mathrm{t}}}{\mu}=0.5 \frac{\rho}{\rho_{\mathrm{b}}}\left(\frac{l_{\mathrm{m}}}{R}\right)^{2} \frac{R e}{U_{\mathrm{b}}}\left|\frac{\partial U}{\partial Y}\right|, \frac{l_{m}}{R}=0.4 Y\left[1-\exp \left(-\frac{y^{+}}{26}\right)\right], y^{+}=0.5 Y \sqrt{\frac{\rho}{\rho_{\mathrm{b}}} \frac{\mu_{\mathrm{b}}}{\mu} \frac{R e}{U_{\mathrm{b}}}}
$$

The Nusselt number $N u$ and heat transfer coefficient $\alpha$ are calculated as:

$$
N u_{\mathrm{b}}=\frac{\alpha d}{\lambda_{\mathrm{b}}}=\frac{q_{\mathrm{w}}}{T_{\mathrm{w}}-T_{\mathrm{b}}} \frac{d}{\lambda_{\mathrm{b}}}=\frac{1}{\Theta_{\mathrm{b}}}, \Theta_{\mathrm{b}}=\frac{\int_{0}^{1} \rho c_{p} U \Theta \eta \mathrm{d} \eta}{\int_{0}^{1} \rho c_{p} U \eta \mathrm{d} \eta}
$$

Solution procedure. Using the finite difference method, the problem of Equations (6)-(8) can be solved with iterative techniques in closed form. The radial domain is discretized into a non-uniform mesh, with the grid compressed near the wall. In the axial direction, a uniform grid of specific enthalpy is adopted. The equations of motion and energy are highly coupled due to the large variations of fluid properties at supercritical pressures, and they are solved iteratively with the updating of fluid properties. All the thermophysical properties of fluid are calculated by calling FORTRAN programs of REFPROP 8.0. Appropriate values of under-relaxation factors for velocity, temperature, turbulence and fluid property variables are chosen to satisfy the convergence criteria.

\section{Results and discussion}

In order to evaluate the present model, numerical simulation has been carried out to predict the cooling heat transfer to turbulent flow of supercritical $\mathrm{CO}_{2}$ in horizontal circular tubes under uniform wall heat flux conditions. The simulation results are compared with the experimental data [3].

Figure 3 shows the effect of mass flux $\left(G=200\right.$ and $\left.400 \mathrm{~kg} \cdot \mathrm{m}^{-2} \cdot \mathrm{s}^{-1}\right)$ on heat transfer coefficient at given parameters $\left(d=6 \mathrm{~mm}, p=8 \mathrm{MPa}, q_{\mathrm{w}}=12 \mathrm{~kW} \cdot \mathrm{m}^{-2}\right)$. The heat transfer coefficient has a peak as the bulk temperature approaches the pseudo-critical temperature where the specific heat achieves its peak value, and it increases with the increasing mass flux similar to the constant-property fluid flows.

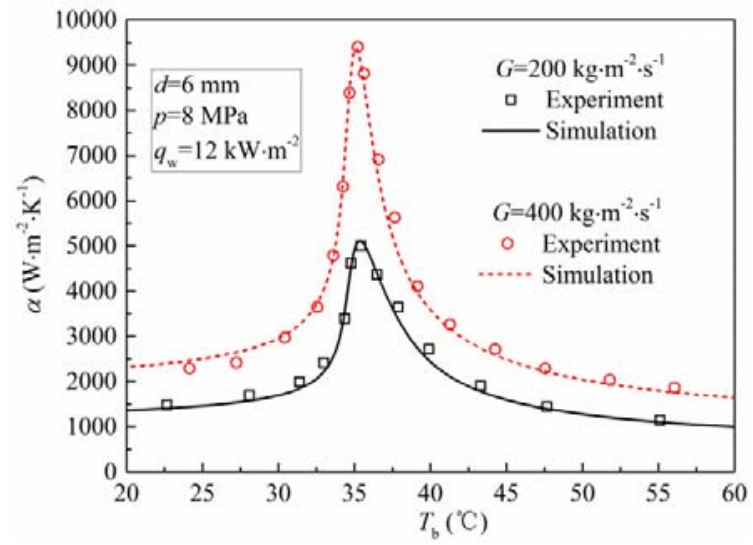

Fig. 3 Effect of mass flux on heat transfer coefficient

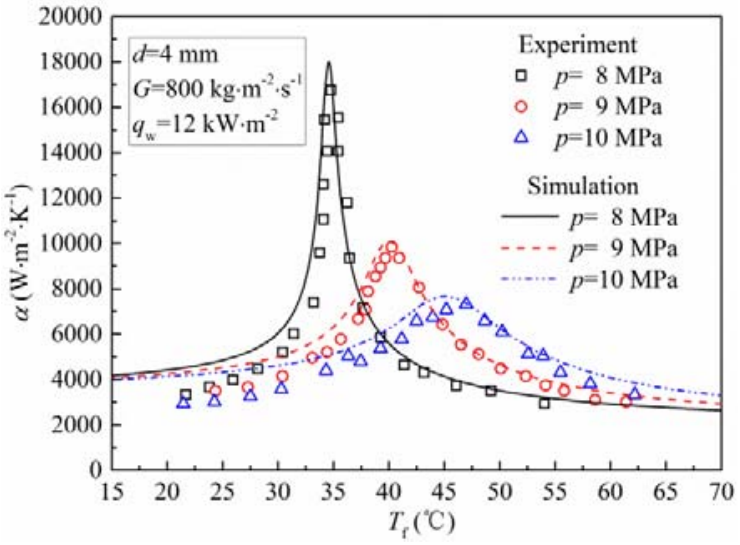

Fig. 4 Effect of pressure on heat transfer coefficient

Figure 4 shows the effect of pressure on heat transfer coefficient at given parameters $(d=4 \mathrm{~mm}$, $\left.G=800 \mathrm{~kg} \cdot \mathrm{m}^{-2} \cdot \mathrm{s}^{-1}, q_{\mathrm{w}}=12 \mathrm{~kW} \cdot \mathrm{m}^{-2}\right)$. According to REFPROP 8.0 , the pseudo-critical temperatures at pressure 8, 9 and $10 \mathrm{MPa}$ are about 34.59, 40.01, and $45.02{ }^{\circ} \mathrm{C}$ respectively. The heat transfer coefficient in the pseudo-critical region increases as the pressure getting closer to the critical pressure, and the maximum occurs approximately where the film temperature $\left(t_{\mathrm{f}}=\left(t_{\mathrm{b}}+t_{\mathrm{w}}\right) / 2\right)$ is equal to the pseudo-critical temperature.

Figure 5 shows the effect of wall heat flux $\left(q_{\mathrm{w}}=6,12,24\right.$ and $\left.33 \mathrm{~kW} \cdot \mathrm{m}^{-2}\right)$ on heat transfer coefficient at given parameters $\left(d=4 \mathrm{~mm}, p=8 \mathrm{MPa}, G=800 \mathrm{~kg} \cdot \mathrm{m}^{-2} \cdot \mathrm{s}^{-1}\right)$. When the bulk temperature $T_{\mathrm{b}}$ is below the pseudo-critical temperature, $q_{\mathrm{w}}$ has less effect on $\alpha$. But when $T_{\mathrm{b}}$ is above the pseudo-critical temperature, $\alpha$ increases obviously with increasing $q_{\mathrm{w}}$. The present simulation results on effect of $q_{\mathrm{w}}$ are in accordance with the FLUENT simulation results by Li et al. [4].

Figure 6 virtually shows the effect of tube diameter $(d=2,4,6 \mathrm{~mm})$ on bulk Nusselt number $\left(N u_{\mathrm{b}}=\alpha d / \lambda_{\mathrm{b}}\right)$ as a function of bulk Reynolds number $\left(R e_{\mathrm{b}}=G d / \mu_{\mathrm{b}}\right)$ at given parameters $(p=8 \mathrm{MPa}$, 
$\left.G \cdot d=2.4 \mathrm{~kg} \cdot \mathrm{m}^{-1} \cdot \mathrm{s}^{-1}, q_{\mathrm{w}}=12 \mathrm{~kW} \cdot \mathrm{m}^{-2}\right)$. It is found that $d$ has slight effect on $N u_{\mathrm{b}}$ when $R e_{\mathrm{b}}$ is fixed.

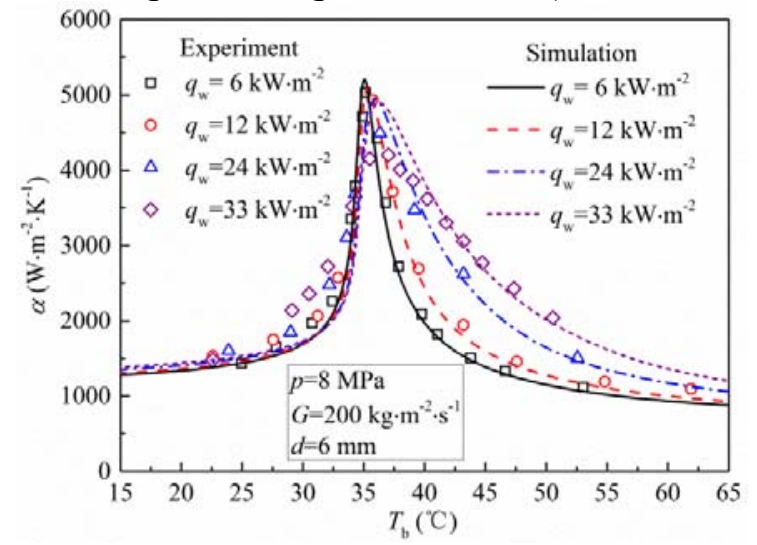

Fig. 5 Effect of heat flux on heat transfer coefficient

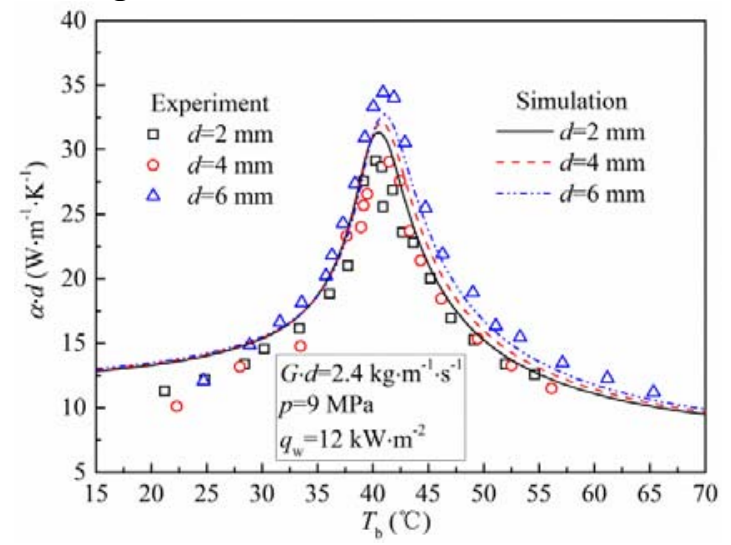

Fig. 6 Effect of tube diameter on heat transfer coefficient

As is shown in Figures 3 6, the simulation results are in good agreement with the experimental data, which indicates that the present model can reproduce the trend of heat transfer characteristics with good prediction performance.

\section{Conclusions}

The problem of turbulent convective heat transfer to supercritical fluids flowing in cooling tubes can be numerically solved using integral boundary-layer equations. With a traditional mixing length turbulence model, the present mathematical model can make fast predictions of the heat transfer coefficient with high accuracy for the enhanced heat transfer regime at supercritical pressures. The simulations results, which are in good agreement with the experimental data, show the mechanism of the effects of mass flux, system pressure, heat flux and tube diameter on heat transfer of supercritical fluid flows.

\section{Acknowledgements}

This research was funded by the National Natural Science Foundation of China (No. 51206040 and 51506043), the Fundamental Research Funds for the Central Universities (No. 2011B11614, 2013B19014 and 2014B19714).

\section{References}

[1] S.H. Yoon, J.H. Kim, Y.W. Hwang, et al., Heat transfer and pressure drop characteristics during the in-tube cooling process of carbon dioxide in the supercritical region, Int. J. Refrigeration 26 (2003) 857-64.

[2] C. Dang, E. Hihara, In-tube cooling heat transfer of supercritical carbon dioxide. Part 1. Experimental measurement, Int. J. Refrigeration 27 (2004) 736-747.

[3] C. Dang, E. Hihara, In-tube cooling heat transfer of supercritical carbon dioxide. Part 2. Comparison of numerical calculation with different turbulence models, Int. J. Refrigeration 27 (2004) 748-760.

[4] H. Li, A. Kruizenga, M. Anderson, et al., Development of a new forced convection heat transfer correlation for $\mathrm{CO}_{2}$ in both heating and cooling modes at supercritical pressures, Int. J. Thermal Sciences 50 (2011) 2430-2442.

[5] H. Tanaka, A. Tsuge, M. Hirata, N. Nishiwaki, Effects of buoyancy and of acceleration owing to thermal expansion on forced turbulent convection in vertical circular tubes, Int. J. Heat and Mass Transfer 16 (1973) 1267-1288. 
[6] E. R. Van Driest, On turbulent flow near a wall, J. Aeronautical Sciences 23 (1956) 1007-1011. 